\title{
A Study on Modeling of Market Circulation of Production Facility Modules by Considering Diversity of Value
}

\author{
Chunyan $\mathrm{WU}^{* *}$ and Akira TSUMAYA**
}

\begin{abstract}
From the perspective of the sustainable manufacturing industry, considering the renovation of manufacturing facilities that are physically and functionally usable is important. This study is based on the hypothesis that the value of the manufacturing facilities is perceived differently among individual users due to differences in production strategies. Therefore, we believe that using a production system composed of a core platform and add-on modules is beneficial, and the long-time use of a core platform can be realized by replacing the add-on modules. Here, the reuse of production facility modules is an important issue. This study proposes a market circulation model of modules considering the diversity of value caused by users' different production plans/strategies. First, a user profile is defined to express the sense of value of each user, such as the user's skills (specializes in certain productions), basic policy on product strategy, preferences, and behavioral principle. Second, to realize a production plan/strategy, a method to derive the user's satisfaction is proposed by comparing the current production system in use with an aspiring production system. When the evaluation of satisfaction is lower than the tolerance level, requirements for changing the current production system can also be calculated. Third, the preference of module trading is modeled as a combinatorial optimization problem of the modules also based on the evaluation of satisfaction. Finally, a multi-agent simulator is constructed consisting of plural user agents with different production strategies in order to evaluate sustainability of entire production facilities in the flower cultivation industry. From the results of the case study, the feasibility of sustainability evaluation is shown.
\end{abstract}

Key words: sustainability, production facility modules, market circulation model, diversity of sense of value, multiagent simulation

\section{Introduction}

As environmental issues have become a global problem, the principle of sustainable development has been proposed ${ }^{1)}$. On the basis of this principle, long-term use of products is demanded from both an economic and an ecological perspective. In terms of production facilities, long-term use is also demanded from the perspective of sustainability. On the other hand, change requests have been made for production facilities to alter products according to changes in market trends or production strategies. Therefore, for a sustainable manufacturing industry, it is important to resolve these conflicting demands.

As a measure for realizing sustainable development, Rose et al. proposed end-of-life strategies based on product characteristics ${ }^{2}$. Their study reported that preferred end-of-life strategies are determined by one of four types of products based on technology cycle and wear-out life:

Type I: Material recovery

Material recovery is a strategy for products that show

\footnotetext{
* Received on May 25, 2015

Accepted on October 29, 2015

Student member, Department of Mechanical Engineering, Graduate School of Engineering, Kobe University (1-1, Rokkodai, Nada, Kobe, 657-8501, Japan) Full member, Department of Mechanical Engineering, Graduate School of Engineering, Kobe University (1-1, Rokkodai, Nada, Kobe, 657-8501, Japan)
}

characteristics of a short wear-out life and a long technology cycle. Type II: Remanufacturing by common parts and modular components

Remanufacturing is a strategy for products that show characteristics of a short wear-out life and a short technology cycle. Type III: Extension of product life by modular design

Extension of product life by modular design is a strategy for products that show characteristics of a long wear-out life and a short technology cycle.

Type IV: Lengthening of product life by maintenance and upgrading capabilities

Lengthening of product life by maintenance and upgrading is a strategy for products that show characteristics of a long wear-out life and a long technology cycle.

The authors categorized manufacturing equipment as type IV and suggested that designers have opportunities to lengthen the product life by facilitating maintenance and simplifying the upgrade process. However, considering the alteration of manufacturing products that corresponds to changing market needs, it appears that updates to production facilities are performed by request in order to update the manufactured product rather than their functional or product life.

Conversely, a product structure that consists of a basic assumption of long-term use and interchangeable module parts has been introduced for some consumer products. The following advantages have been noted in such products: 
1) Response to diverse consumer needs can be realized by increasing the variety of add-on module parts.

2) Cost reductions are expected due to standardization of parts.

3) Various end-of-life strategies can be met through modularization.

In recent years, several studies and proposals have been performed in the research area of product-service systems ${ }^{3) 4}$.

We believe that applying this concept to production facilities can result in both updated facilities due to change requests at the facilities and long-term use of the basic components of the facilities. However, this strategy also leads to the disposal of add-on type modules that are still both functional and have physical life left. Therefore, long-term use through the reuse of obsolete modules is an important issue from the perspective of sustainability. We believe that to solve this, it is necessary to adopt measures that consider long-term business strategies, development of an effective module dividing method, and introduction of a product-service system considering sustainability. However, using these measures to evaluate the sustainability of production facilities in actual world conditions is quite difficult due to their long lifetimes. Therefore, this study proposes a market circulation model for modules and constructs a simulation system based on our proposal.

The purpose of this study is to propose a market circulation model for modules and to construct a simulation system based on the proposed model. As an element that affects the circulation of the modules through buying and selling in the market, we focus on the value differences perceived by individual users. As product requirements for production facilities differ by each producer's production strategy at a given time, and the fact that the condition of used modules sold in the secondhand market are different, we defined a market circulation model for sustainability assessment using simulations and to support a modular design. In this study, we propose a multi-agent market model to evaluate sustainability of manufacturing facilities consisting of a core platform and add-on modules.

\section{Related works}

Much research has been done regarding sustainability to date. Umeda et al. proposed the simulation-based product life cycle design method and simulation system for supporting optimization of a product life cycle design ${ }^{5}$. Kumazawa and Kobayashi proposed the life cycle simulation system that can be evaluated for a transition state from a sales business to a reuse business ${ }^{6}$. Komoto et al. proposed the lifecycle simulation model for analyzing maintenance package design ${ }^{7}$ and also proposed a method to analyze the capability of original equipment manufacturers to satisfy the target of both environmental impacts and lifecycle $\operatorname{costs}{ }^{8}$. This paper focused on the reuse of modules through a second-hand market driven by the differences in the value that individual users feel for the same module. Additionally, numerous studies have been conducted with respect to a module design. Among them, Umeda et al. proposed a methodology that derives a modular structure based on both lifecycle properties and geometric information, and a lifecycle evaluation method of modularized products from the viewpoint of resource efficiency ${ }^{9)}$

${ }^{10)}$. Based on the same idea as such research, this paper introduces the assumption of a production facility consisting of platform parts and add-on module parts.

\section{Concept of market circulation for production facility modules considering value diversity}

In this study, we assume that a production facility is composed of platform parts with long service lives, including a core portion and add-on module parts, which can be replaced with relative ease. The advantages of such a structure are listed in the previous section. Here, a third advantage for consumer products, consisting of a core platform and add-on modules, is identified from the viewpoint of the ability to upgrade. In such cases, the used module that is exchanged is typically no longer of use because of its lack of functional ability. However, when module updating is conducted based on change requests to production facilities, the components to be exchanged have been deemed as low in value by the facility owner, although the end of the product's functional/wear-out life has not yet been reached.

Here, we focus on the differences in the value of module life determined by individual users. Although specifications of the same module such as functionality and physical properties can be described objectively and uniquely, the value perceived by each producer or user of the manufacturing facility is different due to differences in their strategies for product development and production. In addition, the value perceived by an individual user may also differ according to changes in product development and production strategy. Thus, the value of the same module may be low for some users and high for others. Therefore, it is reasonable to suppose that both long-term use of modules and high user satisfaction can be achieved by passing a module from a user who no longer appreciates its value to one who assigns a high value to it. We considered that the transfer of used modules is realized by trading in the secondhand market. Figure 1 shows a conceptual model of module changes by market transactions associated with the changing requirements of the users of the facilities.

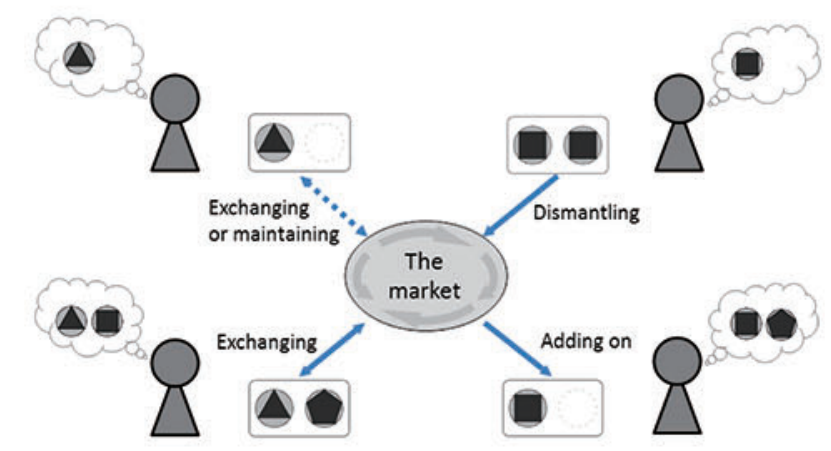

Fig. 1 The concept of the reuse-oriented market model by considering the diversity of values perceived by each user 


\section{Circulation-simulation model of the modules by market transactions considering diversity of value}

\subsection{Overview of the circulation-simulation model}

Based on the concept described above, to evaluate sustainability we constructed a simulation model of the circulation of manufacturing facility modules. By using the simulation model, we considered that the effect of measures to support long-term use of the modules could be evaluated quickly. As the circulation of modules is performed by the trading of modules by the users, the updated activities to modules made by each user are modeled. Each user makes a production plan based on each applicable concept(1).

Next, the user tolerance level of a production facility is determined by the facility's minimum and aspiring levels for achieving the production plan, and the degree of ideal pursued by the user. After that, the tolerance level and current level of the production facility are compared. If the level of their production facility does not satisfy the tolerance level, the causative module will be changed by trading in the secondary market in order to satisfy the user's requirements(2). In this paper, we only reference the users of the production facilities and what they produce in their production facilities, not the "users" who consume their products. The processes of each user's updated module activity are shown in Fig 2.

All users conduct similar activities based on their respective sense of value. The market for modules is formed as a result of these activities. The module manufacturers (makers) supply the module to the market within a range that does not become oversupply(3). The three key points of this model are described in detail below.

\subsection{End-user profile}

Product development and production planning differ for each user because the production planning is determined by market trends and also depends on the characteristics of the individual users.

In this study, all users are given user profiles that represent their characteristics. The user profiles in the simulation are set by considering the following items:

- Expression of the diversity of each user's sense of value.

- Easily generated user agent.

- Easily grasped characteristics of a typical user.

- Expression of a production plan leading to a change in requirement at the production facilities.

By considering the first three items, typical user profiles are created on the basis of many attribute items:

(1) Production skills.

This item indicates a user's skills in producing specified kinds of products. In the simulation, it works to limit the types of products that one user can make.

(2) Basic policy on product strategy.

This item details a user's behaviors such as:

i. Under the limitation of item (1), does the user produce single or multiple kinds of products?

ii. Does the user ever change what used to be produced?

iii. Except for normal production, does the user take a new order or not?

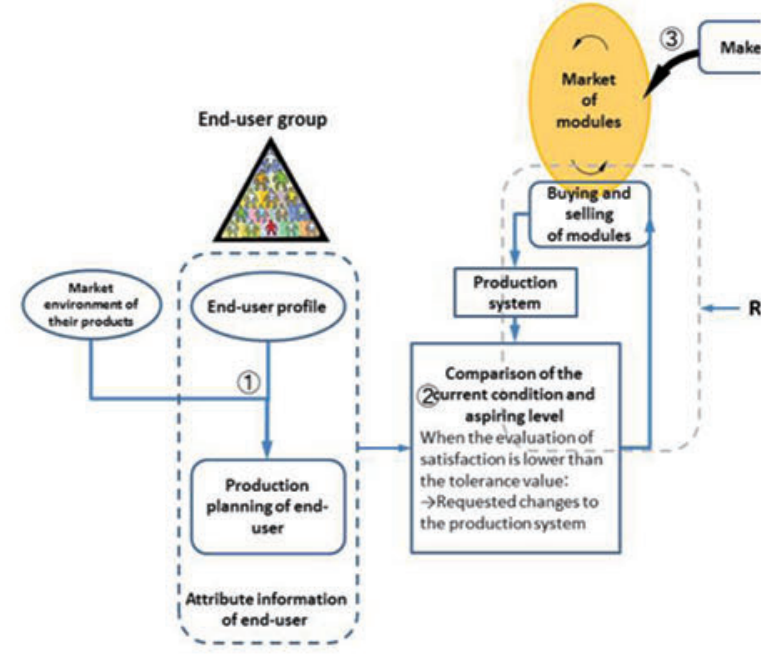

Fig. 2 The concept of updating modules through the market

iv. Does the user change the production plan in the short-term, mid-term, or long-term?

v. Does the user have a tendency to produce a small, medium, or large production quantity?

Such items define the user profile in a broad outline, but there are still many other elements that have more or less influence on the user's decision making.

(3) Company size.

This item includes details that describe the user's business station, such as:

i. Producing capability, maximum of production.

ii. Budget for investments.

(4) Whether or not the user is a risk taker.

When a significant benefit comes with a higher risk, does the user have the tendency to choose the risky decision?

(5) Whether or not the user is sensitive to fashion trends.

Does the user pick up on information about hot or rare goods?

(6) Whether or not the user is changeable.

When there are fluctuations in the market, does the user have a tendency to review and change the production plan in the short-term?

(1) is a limiting item that plays a decisive role. Moreover, (2) is an item that generally divides users into many classifications. (3) is a less important restriction item. (4) through (6) are items of uncertain influence. Examining these items, a user's profile can be described in general at first, and then be detailed by some uncertain differences in order to indicate user diversity based on different attribute items, from an abstract level to a detail specific level.

On the other hand, consider the market environment elements of the user's products:

(1) Order information.

This item influences a user's production of orders.

(2) Hot good(s).

(3) Rare good(s).

If a user is a risk taker, when information for hot goods 
or rare goods comes with the potential of greater benefit gain, the user may readily change the production plan to a plan for these hot products.

(4) Well-selling line over the past years.

This item may be useful to users who regularly create their production plans in the mid- or long-term.

From typical user profiles and market environment elements, product strategy, such as the decision to produce certain items, can be presumed by a rule-based method. Furthermore, the length of the production planning can be presumed by judgments such as how often a user makes a decision. In the same way, the number of production items and their production processes, and the production volume of each production item are added to the user profile in consideration of the final item.

4.3 Change requests for production facilities due to changes in production plan

As noted above, the production plan is determined regularly from the market environment and the user profile. In this process, the production plan is often altered. The following objectives can be cited as typical for changes in production planning.

- To change production items.

- To produce higher quality products.

- To expand production items.

- To decrease production items.

- To simplify the production system.

- To compensate for failure of function.

Following the determination of the new production plan, the aspiration level $U L a$ and the tolerance level $U L t$ are determined to ideally satisfy the requests of production facilities and the minimum level $U L m$ required for producing (a) target production item(s) is also determined. Of course, $U L m \leq U L t \leq U L a$. Here, the user must judge whether the condition of the production facility in current use, $U L c$, can satisfy the requests by comparing $U L c$ with $U L m, U L t$, and $U L a$.

There are many reasons and limiting conditions affecting a user's final decision on selecting a module or a module set. First, the function level $f_{m, i}$ and remaining life $r_{m, j}$ are introduced as the two main items for a user to evaluate module $m$ in current use:

$f_{m, i}$ : Function level of function $i$ of module $m$.

$r_{m, j}$ : Value of factor $j$ that affects the remaining life of the module $m$.

Using these definitions, the state of module $\mathrm{m}$ in current use $\mathrm{s}_{\mathrm{m}}$ can be defined as:

$$
s_{m}=\left[f_{m, 1}, f_{m, 2} \cdots, f_{m, k}, r_{m, 1}, r_{m, 2} \cdots r_{m, l}\right]
$$

where subscript $k$ is the total number of the function type, and subscript $l$ is the total number of the factors that affect the remaining life.
Here, when the new production plan is decided, the aspiration level of the facility $U L a$ and the minimum level of the facility for production $U L m$ are given as follows:

$$
\begin{gathered}
U L m=\left[U_{\min , 1}, U_{\min , 2}, \cdots, U_{\min , k}\right] \\
U L a=\left[U_{a, 1}, U_{a, 2}, \cdots, U_{a, k}\right]
\end{gathered}
$$

Module evaluating can be performed by determining its degree of satisfaction as an index, including the following criteria:

- The case below minimum level $U_{m i n, i}$ is described as 0 .

- The case above aspiring level $U_{a, i}$ is described as 1 .

- The case between minimum level $U_{\min , i}$ and aspiring level $u_{a, i}$ is described as a monotonically increasing continuous function or as a discrete value sequence that approximates this function.

Next, the utility value of each function the module contains and the features reflecting the remaining life can be calculated by the following formula.

$$
\begin{gathered}
u_{m, i}=g_{i}\left(f_{m, i}\right) \cdot h_{i}\left(r_{m, j}\right) \\
u_{m}=\left[u_{m, 1}, u_{m, 2} \cdots, u_{m, k}\right]
\end{gathered}
$$

where $g_{i}\left(f_{m, i}\right)$ is a function in accordance with the above criteria, and $h_{i}\left(r_{m, j}\right)$ is a function that is determined by the feature of function degradation, the mean time to failure and its derivation, and the opinion of the user.

As previously mentioned, we assumed that a production facility is composed of platform parts and add-on module parts. Since the evaluation of the production facility is highly dependent on the evaluation of its components, we derive the evaluation value of the production facility from the utility value of the composed modules. Here, following the two assumptions introduced above, each function is independent of each other, and there are no synergistic effects about the level of function by a plurality of the modules. Under these assumptions, the evaluation value of the production facility in current use can be defined in the following formulas:

$$
\begin{gathered}
U_{c, i}=\max _{m} u_{m, i} \\
U L C=\left[U_{c, 1}, U_{c, 2} \cdots, U_{c, k}\right]
\end{gathered}
$$

$U L c$ : A total index to describe the state of production facility in current use.

Because of the diversity of values, ULt depends on the user's many decision-making attributes in pursuing high-level or long remaining life of modules. We suggest it depends on the user's profile as well, and it can be decided by analyzing user's consumption activity in the real world.

$$
U L t=\left[U_{t, 1}, U_{t, 2}, \cdots U_{t, k}\right]
$$

Next, by comparing the aspiration level $U L a$, the tolerance level $U L t$, and the minimum level Ulm of the required production facilities with the level of production facilities in current use $U L c$, it 
Table 1 Trading module activities based on user production strategy

\begin{tabular}{|c|c|c|c|c|c|}
\hline \multicolumn{2}{|c|}{ Objectives } & \multirow{2}{*}{$\begin{array}{l}\text { Evaluation } \\
\boldsymbol{U}_{c, i}<\boldsymbol{U}_{t, i}\end{array}$} & \multirow{2}{*}{$\begin{array}{c}\begin{array}{c}\text { Purcha } \\
\text { ses }\end{array} \\
0\end{array}$} & \multirow{2}{*}{$\begin{array}{c}\text { Sales } \\
\mathrm{O}\end{array}$} & \multirow{2}{*}{\begin{tabular}{|c|} 
Wastes \\
0 \\
\end{tabular}} \\
\hline $\begin{array}{l}\text { To change the } \\
\text { production } \\
\text { item }\end{array}$ & Function change & & & & \\
\hline $\begin{array}{l}\text { To produce } \\
\text { higher quality } \\
\text { products }\end{array}$ & $\begin{array}{l}\text { Upgrade } \\
\text { (Enhancing, } \\
\text { Addition) }\end{array}$ & $\begin{array}{c}U_{t, i \text { as is }} \\
< \\
U_{t, i \text { to be }}\end{array}$ & $\mathrm{O}$ & & $\mathrm{O}$ \\
\hline $\begin{array}{l}\text { To expand the } \\
\text { production } \\
\text { item }\end{array}$ & $\begin{array}{l}\text { Upgrade } \\
\text { (Enhancing, } \\
\text { Addition) }\end{array}$ & $\boldsymbol{U}_{c, i}<\boldsymbol{U}_{t, i}$ & $\mathrm{O}$ & & $\mathrm{O}$ \\
\hline $\begin{array}{c}\text { To decrease } \\
\text { the } \\
\text { production } \\
\text { item }\end{array}$ & $\begin{array}{l}\text { Function } \\
\text { reduction }\end{array}$ & $\boldsymbol{U}_{c, i}>\boldsymbol{U}_{a, i}$ & & 0 & 0 \\
\hline $\begin{array}{l}\text { To simplify } \\
\text { the system }\end{array}$ & $\begin{array}{l}\text { Function } \\
\text { reduction }\end{array}$ & $\boldsymbol{U}_{c, i}>\boldsymbol{U}_{a, i}$ & & O & 0 \\
\hline $\begin{array}{l}\text { A failure of } \\
\text { function }\end{array}$ & $\begin{array}{c}\text { Function } \\
\text { maintenance }\end{array}$ & $U_{c, i}<U_{\min , i}$ & $\mathrm{O}$ & $\mathrm{O}$ & $\mathrm{O}$ \\
\hline
\end{tabular}

is possible to calculate the presence of change requests to the production facilities.

Cases when $U L c$ reaches $U L a$ or just above it a little bit, there are no change requests to the production facilities. Cases when $U L c$ is far greater than $U L a$, the user tends to simplify the facility by selling some modules. However, in cases when $U L c$ is below $U l m$ or $U L t$, change requests happen. According to the change request, as an inevitable result of exchanging or simplify modules of a facility, modules that seem to no longer be used (and be removed) by a user will come out. We suppose each user will dispose some of them or perform trading activity with some of them on the module market. Table 1 shows trading modules based on changes in production planning.

\subsection{Preferences of modules in trading activities}

In this study, because the candidate modules are finite, the selection of preferred modules is treated as a combinatorial optimization problem that considers the user satisfaction for each evaluated item. Here, we consider that the user's degree of satisfaction and the value perceived by the user is equivalent. The formulation of the method is described as follows and is described as a utility.

Suppose there is a set $M$ of a certain type of module in the market, and module $\mathrm{m}$ belongs to set $M$. Under the budget constraints, the user's activity of selecting a target module or a set of modules in the market is performed as: First, calculating the utility value of all modules in the market at the present time, and searching all of the modules that satisfy index $U_{t, x}$. In some cases, if there is no option in the market at the present better than what is in current use, the user will give up these purchasing activities and maintain the current situation of their production facility. Then, for each candidate of the module (or set of modules) to be purchased, the total utility value defined by the following formula is calculated,

$$
U_{p}=w_{1} u_{p, 1}+w_{2} u_{p, 2}+\cdots+w_{k} u_{p, k}+w_{k+1} c_{p}
$$

where $c_{p}$ is the purchase cost of the candidate, subscript $p$ is a unique ID attached to each of the candidates, and $w_{k}$ is the weight

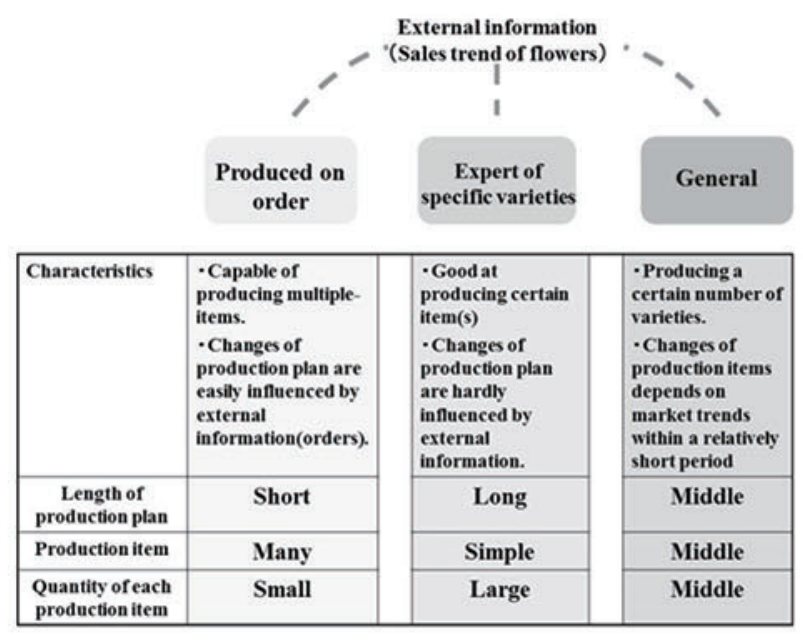

Fig.3 Classification table of users in case study

of each evaluation $\left(w_{1}+w_{2}+, \cdots,+w_{k+1}=1\right)$. Finally, the candidate module (or set of modules) that has the highest utility value $\max _{p} U_{p}$ will be selected for purchase.

The user's decision-making processes and parameter changes in the market are shown below.

\section{Implementation and case study}

On the basis of the previously described model, a multi-agent simulator was implemented consisting of plural user agents, a maker agent, and a waste collector agent for reproducing the circulation of modules through the module market. There are no special dealings differ from a normal multi-agent modeling method employed in our research.

In this study, an automatic production system for flower cultivation was adopted as a case study. For the user profile, we classified users into the following three types (Fig.3) on the basis of interviews with the flower producers in Yunnan Province, China, one of the main flower production districts in the world.

- Produced on order: This type represents large-scale producers that can support the production of a wide variety of flowers. Changes in production items depend on the status of orders.

- Expert of specific varieties: This type of producer specializes in the production of specific varieties of flowers.

- General: This type represents producers of a certain number of varieties. Although production items are few, there is a certain level of flexibility in production. This type usually makes changes to the production items according to market trends within a relatively short period.

Module parts of manufacturing facilities were classified as five types by functionality $\left(F_{1}, F_{2}, F_{3}, F_{4}, F_{5}\right)$ and five stages of quality for each functional type $\left(L_{1}, L_{2}, L_{3}, L_{4}, L_{5}\right)$. Thus, a total of 25 types of modules were set. The designed life of all modules is supposed as 10 years. The varieties of the production items (flowers) were set as four topic types A, B, C, and D. 
Each user agent production plan was determined on the basis of the user profile; the skills represent each type of flower, length of the production planning, and degree of follow-up with market trends. For the phase deciding the preferred module, the minimum production facility level represents the fixed level required for handling the production of each type of flower.

As noted in Section 4.3, the aspiring level is determined by each user's production strategy. To determine the preferred module, the values of the following three items are referred to for evaluation: price of module, quality stage of module function, and its anticipated residual service life.

A case study is presented that uses the simulator implemented in our proposed model. The number of users was set to 1000 people, and the length of the simulation period was set to 50 years. The simulation was performed for the three scenarios described below:

- Scenario 1: The module is discarded when it is deemed valueless by the user.

- Scenario 2: When the user has determined that the module in valueless, the user decides whether or not to discard or sell the module according to its condition.

- Scenario 3: Considering the assumption of Scenario 2, activities such as inspection and repair by the recovery operators are added.

The activities of the agents are reflected as rewritten numerical values of the modules. For example, the activity "use" of the user agent changes to the rewritten numerical value of the module attribute item "used year," and the activities of the waste collector are rewritten numerical values of module attribute items such as "residual life," "resale price," and "service history," of the modules. We analyze the results by totaling the numerical values of every item.

Figures 4,5 , and 6 show the results of usage age distribution through 50 years for Scenarios 1, 2, and 3, respectively. The total number of discarded modules is significantly reduced in Scenario 3. In addition, for Scenario 3 and Scenario 2, the distribution of usage age also shifts toward longer periods. Therefore, when qualities such as function and residual service life are secured, modules can be reused according to the diversity of the perceived value of each user. This result shows the feasibility of long-term use of modules on the core platforms and the add-on modules of the production facility. The results of the case study also show that it is possible to evaluate the sustainability of modules of production facilities by using our proposed model.

The reasons why a peak appears around years nine and 10 are as follows. One is the modules' designed lifetime. In this case study, we set designed lifetime of all modules as 10 years. The other reason is timing of users' production re-planning. In this case study, every user set the period of production plan between two and five years. Therefore, the first large peak appears at the first and fifth year and the second appears in the years that reach the condition where the end of the next production plan is the designed lifetime.

In Scenario 3, inspection and repair processes are introduced to the modules in the secondhand market. We handled the effect of the

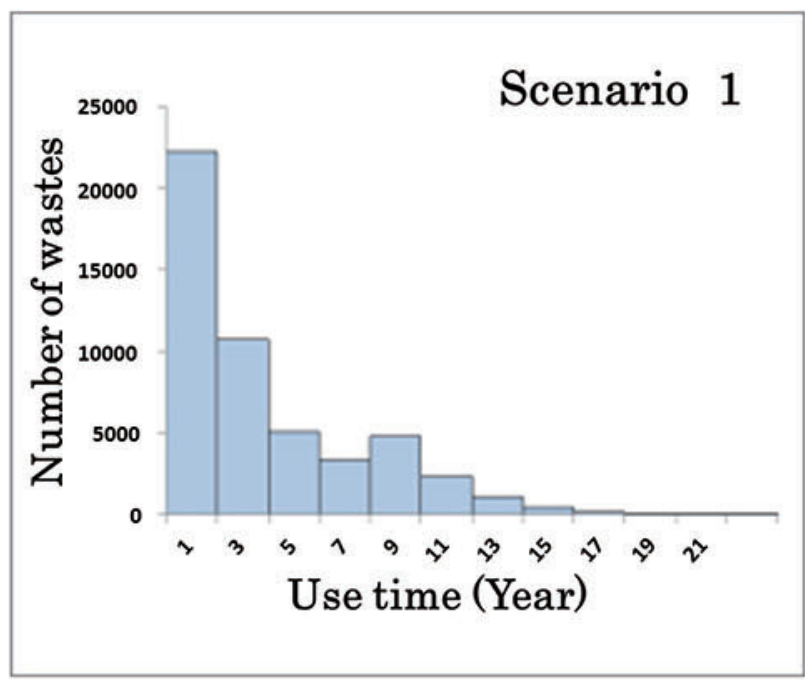

Fig. 4 Distribution of age of usage through 50 years for Scenario 1

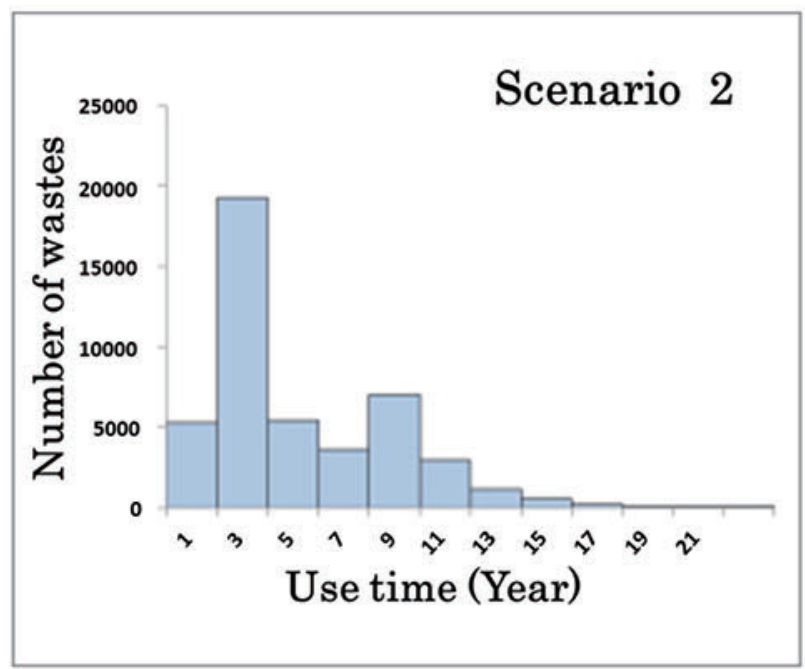

Fig. 5 Distribution of usage age through 50 years for Scenario 2

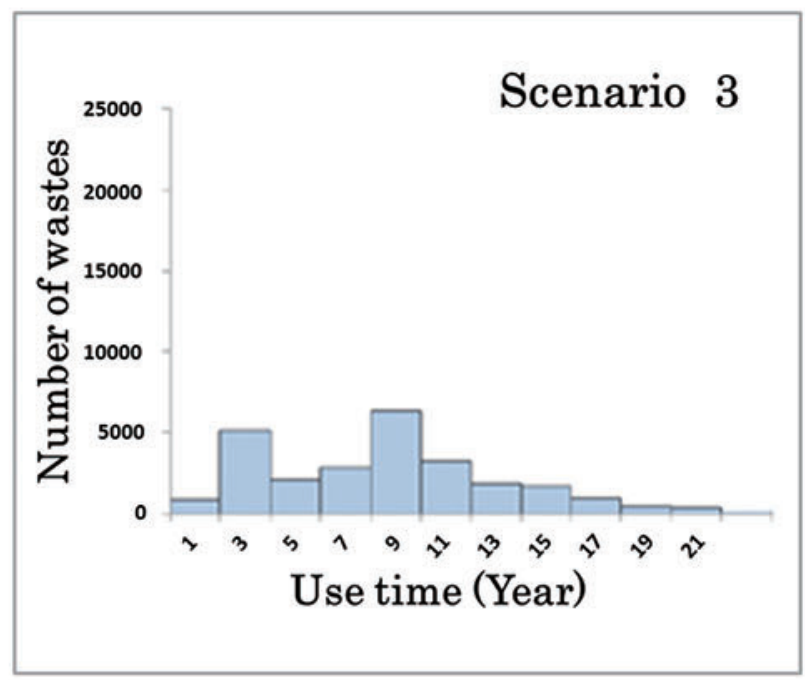

Fig. 6 Distribution of usage age through 50 years for Scenario 3 
introduction of these processes as follows:

- Remaining lifetime of the module is slightly increased.

- Reliability of remaining lifetime of the module is improved.

- Price of the module is higher because the inspection and repair costs are added.

The result of the simulation in Scenario 3 suggests that introducing inspection and repair processes leads to long-term use due to reuse of the module. In the same way, measures such as introducing maintenance service or the adjustment of the quantitative balance of a new product market and secondhand product market can lead to long-term use of the modules. Although numerous trials and errors are needed to consider these measures, our proposed simulation system can provide a virtual environment for evaluating them.

The proposed model is focused on products that belong to type IV, which are characterized by long technology cycles and long wearout life. Therefore, the market circulation model constructed by the proposed method can also be expected to be effective for evaluating type IV products such as types of machine modules.

\section{Conclusion}

From the perspective of the sustainable manufacturing industry, it is important to consider renovations of manufacturing facilities that are physically and functionally useful. In this study, the concept of a module circulation model is proposed considering perceived value diversity of each user. This concept is based on the hypothesis that the value of the manufacturing facilities is different to individual users due to differences in their production strategies. Thus, a circulationsimulation model of modules according to market transactions that consider value diversity was proposed. In this paper, three key processes of the model were described in detail. First, a model description of the user profile was defined to express the sense of values of each user. Second, a method for user requirements was derived to determine changes to production facilities, based on differences in the desired production planning/strategies and the existing production systems. Third, the preference for module trading was modeled as a combination optimization problem of the modules based on evaluation of perceived satisfaction. By using a multi-agent simulation system implemented based on the proposed model, a case study was examined. According to the results of the simulations, our proposed system can be suggested as an effective way to perform sustainability evaluation from the perspective of the long-term use of modules.

\section{References}

1) United Nations: Report of the World Commission on Environment and Development, General Assembly Resolution 42/187, (1987).

2) C. M. Rose, K. A. Beiter, K. Ishii, and K. Masui: Characterization of Product End-of life Strategies to Enhance Recyclability, Proc. DETC98 ASME Design for Manufacturing Symposium, DETC98/DFM-5742, (1998).

3) C. Mannweiler and J.C. Aurich: Modularization of Products and Services for Configuring Product-Service Systems, Y. Shimomura and K. Kimita (Eds.) The Philosopher's Stone for Sustainability, Springer, (2012)37.

4) H. Hiroka, T. Ueno, M. Arita, Y. Shigeji, K. Horii, and H. Kawaharada: Promoting reuse of mechanical parts using part agents, Y. Shimomura and K. Kimita (Eds.) The Philosopher's Stone for Sustainability, Springer, (2012)387.

5) Y. Umeda, A. Nomura, and T. Tomiyama, Study on Life-cycle Design for the Post Mass Production Paradigm, Artificial Intelligence for Engineering Design, Analysis and Manufacturing, 14, (2000)149.

6) T. Kumazawa and H. Kobayashi, A Simulation system to support the establishment of circulated business, Advanced Engineering Informatics, 20, (2006)127.

7) H. Komoto and T. Tomiyama, Design of Competitive Maintenance Service for Durable and Capital Goods Using Life Cycle Simulation, Int. J. Automation Technology, 3, 1, (2009) 63.

8) H. Komoto, T. Tomiyama, S. Silvester, and H. Brezet, Analyzing Supply Chain Robustness for OEMs from a Life Cycle Perspective Using Life Cycle Simulation, Int. J. Production Economics, 134, (2011)447.

9) Y. Umeda, S. Fukushige, K. Tonoike, S. Kondoh, Product modularity for life cycle design., CIRP Annals-Manufacturing Technology 57, (2008)13.

10) G. Seliger, M. Zettl, Modularization as an enabler for cycle economy, CIRP Annals-Manufacturing Technology 57, (2008)133. 